\title{
Task force launching to examine national licensure for virtual care
}

\author{
Cite as: CMAJ 2019 April 15;191:E429. doi: 10.1503/cmaj.1095738
}

Posted on cmajnews.com on Mar. 26, 2019.

$\mathbf{N}$ ational medical organizations are investigating barriers to virtual care in Canada, as well as the feasibility of national licensure.

The Canadian Medical Association (CMA), Royal College of Physicians and Surgeons of Canada, and the College of Family Physicians of Canada are launching a task force to explore what regulatory changes are needed to allow doctors to deliver telemedicine across provincial and territorial borders. It will also examine how telemedicine can improve access and quality of care, as well as ways to capture and share health information.

"It is time for our policies and regulations to evolve to today's available technology," according to CMA President Dr. Gigi Osler. The task force will involve representatives from regulatory bodies, medical and health care groups and the eHealth industry. It will issue recommendations by early 2020 .

Seven out of 10 Canadians say they would take advantage of virtual physician visits if they were available, according to a 2018 Ipsos poll. Telemedicine programs have demonstrated cost savings and improvements in patient outcomes and access to care. These programs have taken off in the United States, but in Canada, administrative and financial barriers remain.

According to the Canadian Medical Protective Association, in the absence of a national framework for telemedicine, licensing requirements for physicians vary across the country. Some physicians providing virtual care might need to be licensed in two jurisdictions: their own and the patient's.

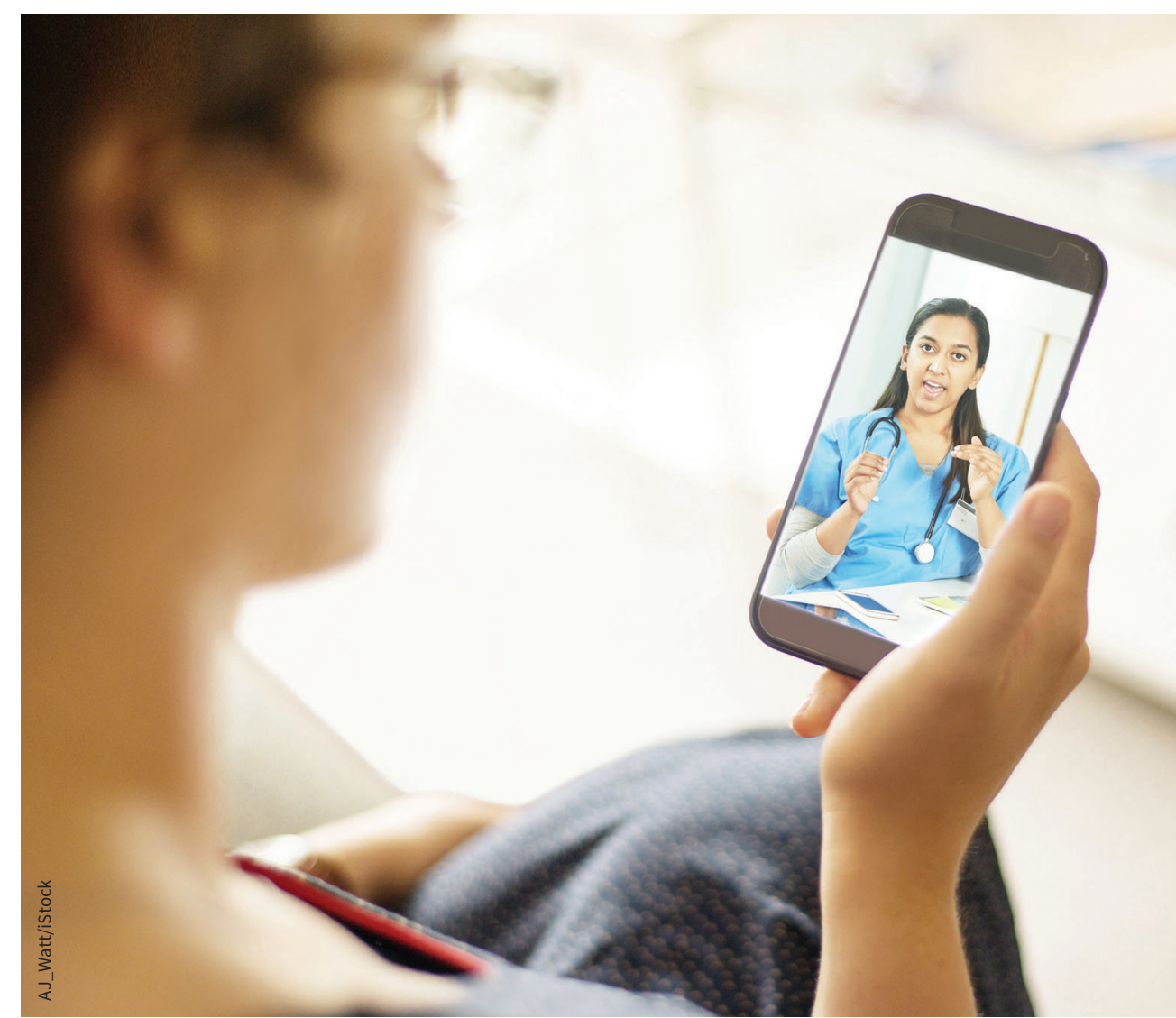

Differences in physician licensure and regulation across Canada have slowed the uptake of telemedicine.

Most regulatory colleges have published telemedicine bylaws or policies, and some jurisdictions may require special registration or place conditions on the provision of virtual care. For example, some colleges impose additional consent requirements for telemedicine services.

Privacy rules also vary across the country, posing a challenge to sharing health information across jurisdictions. Meanwhile, public reimbursement for vir- tual care remains patchy. A growing number of private telemedicine companies are filling the void, raising concerns about the universality of virtual care.

According to Dr. M. Ian Bowmer, president of the Royal College, "this is a timely opportunity to improve access and communication between patients and their doctors."

Lauren Vogel, CMAJ 